\title{
LOCAL GEVREY AND QUASI-ANALYTIC HYPOELLIPTICITY FOR $\square_{b}$
}

\author{
BY DAVID S. TARTAKOFF 1
}

Communicated by Daniel W. Stroock, May 27, 1976

Introduction. The $\bar{\partial}_{b}$ complex is well defined on any smooth $C R$ manifold $M$, and once a metric is fixed, so is the complex Laplace-Beltrami operator $\square_{b}$ on forms of type $(p, q)$. For compact $M$ without boundary, the $\bar{\partial}_{b}$ cohomology of $M$ may be studied via $\square_{b}$ [6], and thus local smoothness of solutions to $\square_{b} u$ $=f$ is important. In its own right, $\square_{b}$ is a prototype of doubly characteristic operators. Under suitable convexity conditions on $M$, Kohn [7] established the following subelliptic estimate on $(p, q)$ forms in $C_{0}^{\infty}(M)$ :

$$
\|\varphi\|_{1 / 2}^{2} \leqslant C\left(\square_{b} \varphi, \varphi\right)+C^{\prime}\|\varphi\|_{0}^{2},
$$

and in general such estimates imply $C^{\infty}$ and Gevrey $\left(G^{s}, s \geqslant 2\right)$ hypoellipticity locally [3] , [8], [10] and no more [1]. In the special case of the Heisenberg group, Folland and Stein [5] found an explicit fundamental solution which gives local analytic hypoellipticity; while, in general, if $M$ is compact, satisfies the convexity condition $Y(q)$ of Kohn, and has an invertible Levi form, the author proved $\square_{b}$ is globally analytic hypoelliptic and so is the $\bar{\partial}$-Neumann problem (joint work with M. Derridj, cf. [4], [9]).

In this note we assume $Y(q)$ and the invertibility of the Levi form and prove local regularity in all Gevrey classes $G^{s}$ with $s>1$ as well as in a quasianalytic class. Full details will appear elsewhere.

Notations and definitions. The class $C^{L}(\Omega) \subset C^{\infty}(\Omega), \Omega$ open in $R^{n}$, is defined by the condition that for all $K \subset \subset \Omega$ there exists a constant $C_{f, K}$ such that for any multi-index $\alpha$,

$$
\sup _{K}\left|D^{\alpha} f\right| \leqslant C_{f, K}^{|\alpha|+1} L(|\alpha|)^{|\alpha|}
$$

where we assume that the sequence $\{L(j)\}$ of positive numbers satisfies $(1) L(j) / j$ is nondecreasing and (2) $L(j+1)^{j+1} \leqslant C^{j} L(j)^{j}$ uniformly in $j$. The second condition implies that $C^{L}(\Omega)$ is closed under differentiation while the first implies that the class is preserved under composition. Thus one may speak of $C^{L}$ manifolds. If, in addition, $\Sigma L(j)^{-1}<\infty$, the class is called non-quasi-analytic (NQA) and admits compactly supported functions. Common examples are the Gevrey classes $G^{s}(\Omega)$, obtained by taking $L(j)=j^{s}$. These are NQA if $s>1$, while 
taking $s=1$ gives the real analytic class. The class obtained by taking $L(j)=$ $j \log j$ is quasi-analytic, and thus contains no compactly supported functions.

A smooth manifold $M$ of dimension $2 n-1$ is called $C R$ provided (a) $\mathrm{C} T(M)_{x}=S_{x} \oplus \overline{S_{x}} \oplus F_{x}$ for all $x, S$ a smooth subbundle of $\mathrm{C} T(M)$ of complex dimension $n-1$, orthogonal to $\bar{S}$ under a smooth Hermitian inner product which induces a Riemannian metric on $M$, and $F$ of complex dimension 1 , and (b) if $Y_{1}, Y_{2}$ are local sections of $S$, so is their commutator $\left[Y_{1}, Y_{2}\right] . D^{p, q}(M)$, the space of smooth $(p, q)$ forms on $M$, is defined to be the space of those smooth $p+q$ forms on $M$ such that $h\left(t_{1}, \ldots, t_{p+q}\right)=0$ if $p$ of the $t$ 's and $q$ of the $\bar{t}$ 's have zero projection on $S$. For $v \in D^{p, q}(M)$ we define $\bar{\partial}_{b} v$ to be the projection on $D^{p, q+1}(M)$ of $d v$. The $\bar{\partial}_{b}$ form a complex and we denote by $\square_{b}$ : $D^{p, q}(M) \rightarrow D^{p, q}(M)$ the operator $\bar{\partial}_{b} \bar{\partial}_{b}^{*}+\bar{\partial}_{b}^{*} \bar{\partial}_{b}$, where $\bar{\partial}_{b}^{*}$ denotes the formal $L^{2}$ adjoint of $\bar{\partial}_{b}$. When $Y_{1}, \ldots, Y_{n-1}$ forms a local frame for $S$ and $T$ denotes a local, nowhere zero, purely imaginary section of $F$, the matrix $c_{i j}$, given by $\left[Y_{i}, \bar{Y}_{j}\right] \equiv c_{i j} T$ modulo $S \oplus \bar{S}$, is the Levi form of $M$. The number of its nonzero eigenvalues and its signature in absolute value are independent of the choice of $Y_{j}$ and of $T$. $M$ satisfies $Y(q)$ if $c_{i j}$ has $\max (q+1, n-q)$ eigenvalues of the same sign or pairs of eigenvalues of opposite signs. $M$ is strictly pseudoconvex if all eigenvalues are strictly of the same sign.

\section{Results.}

THEOREM. Let $M$ be a $C R$ manifold of class $C^{L}$, L satisfying (1) and (2) above and non-quasi-analytic, of dimension $2 n-1$ with an invertible Levi form satisfying $Y(q)$ in an open set $\Omega$. Then any $u \in D^{p, q}(\Omega)$ satisfying $\square_{b} u \in$ $C^{L}(\Omega)$ is itself in $C^{L}(\Omega)$.

Proposition (Boman, CF. [2]). The intersection of all $C^{L}(\Omega)$, L satisfying (1) and (2) and non-quasi-analytic, is the class $C^{L^{\prime}}$ where $L^{\prime}(j)=j \log j$.

COROLlARY. Let $M$ be a CR manifold of the quasi analytic class $C^{L^{\prime}}$, $L^{\prime}(j)=j \log i$, with invertible Levi form satisfying $Y(q)$. Then any $u \in D^{p, q}(\Omega)$, $\Omega$ open in $M$, with $\square_{b} u \in C^{L^{\prime}}(\Omega)$, is itself in this class.

Remarks. It is well known that one need only assume that $u \in D^{\prime}(\Omega)$ for the above results to hold [6] , [8]. Also, there is a direct proof of the Corollary which obviates the quasi-analyticity of $C^{L^{\prime}}$ and obtains the local result by considering a family of compactly supported functions whose derivatives, up to a given order, grow uniformly as if the functions belonged to $C^{L^{\prime}}$. Families of this sort were introduced by Ehrenpreis to localize some real analytic problems; while his families fail to satisfy an analogue of (1) above, one may approximate $C^{L^{\prime}}$ by families which do.

\section{REFERENCES}

1. M. S. Baouendi and C. Goulaouic, Analyticity for degenerate elliptic equations and 
applications, Proc. Sympos. Pure Math., vol. 23, Amer. Math. Soc., 1973, pp. 79-84. MR 50 \#5167.

2. J. Boman, On the intersection of classes of infinitely differentiable functions, Ark. Mat. 5 (1963/65), 301-309. MR 36 \#116.

3. M. Derridj, Sur la régularité $G^{2}$ au bord des solutions du problème de Neumann pour l'opérateur $\bar{\partial}$ (to appear).

4. M. Derridj and D. S. Tartakoff, On the Global Real Analyticity of Solutions to the $\bar{\partial}$ Neumann Problem, Comm. in Partial Differential Equations.

5. G. B. Folland and E. Stein, Estimates for the $\bar{\partial}_{b}$-complex and analysis on the Heisenberg group, Comm. Pure Appl. Math. 27 (1974), 429-522. MR 51 \#3719.

6. J. J. Kohn, Harmonic integrals on strongly pseudo-convex manifolds. I, II, Ann. of Math. (2) 78 (1963), 112-148; ibid. 79 (1964), 450-472. MR 27 \#2999; 34 \#8010.

7. - Boundaries of complex manifolds, Proc. Conf. Complex Analysis, (Minneapolis, 1964), Springer-Verlag, Berlin, 1965, pp. 81-94. MR 30 \#5334.

8. D. S. Tartakoff, Gevrey hypoellipticity for subelliptic boundary value problems, Comm. Pure Appl. Math. 26 (1973), 251-312. MR 49 \#7586.

9. - On the global real analyticity of solutions to $\square_{b}$ on compact manifolds, Comm. in Partial Differential Equations (to appear).

10. F. Treves, An invariant criterion of hypoellipticity, Amer. J. Math. 83 (1961), 645-668. MR 24 \#A2732.

SCHOOL OF MATHEMATICS, INSTITUTE FOR ADVANCED STUDY, PRINCETON, NEW JERSEY 08540 